\title{
A NickelBisdiamine Porous Organic Polymer as Heterogeneous Chiral Catalyst for Asymmetric Michael Addition to Aliphatic Nitroalkenes
}

\author{
Buendia, Mikkel Burggraaf; Kegnæs, Søren; Kramer, Søren
}

Published in:

Advanced Synthesis and Catalysis

Link to article, DOI:

10.1002/adsc.202000875

Publication date:

2020

Document Version

Peer reviewed version

Link back to DTU Orbit

Citation (APA):

Buendia, M. B., Kegnæs, S., \& Kramer, S. (2020). A NickelBisdiamine Porous Organic Polymer as Heterogeneous Chiral Catalyst for Asymmetric Michael Addition to Aliphatic Nitroalkenes. Advanced Synthesis and Catalysis, 326(23), 5506-5512. https://doi.org/10.1002/adsc.202000875

\section{General rights}

Copyright and moral rights for the publications made accessible in the public portal are retained by the authors and/or other copyright owners and it is a condition of accessing publications that users recognise and abide by the legal requirements associated with these rights.

- Users may download and print one copy of any publication from the public portal for the purpose of private study or research.

- You may not further distribute the material or use it for any profit-making activity or commercial gain

- You may freely distribute the URL identifying the publication in the public portal 


\section{Advanced $\Rightarrow$ Synthesis \& Catalysis}

\section{Accepted Article}

Title: A Nickel-Bisdiamine Porous Organic Polymer as Heterogeneous Chiral Catalyst for Asymmetric Michael Addition to Aliphatic Nitroalkenes

Authors: Mikkel Buendia, Søren Kegnæs, and Soren Kramer

This manuscript has been accepted after peer review and appears as an Accepted Article online prior to editing, proofing, and formal publication of the final Version of Record (VoR). This work is currently citable by using the Digital Object Identifier (DOI) given below. The VoR will be published online in Early View as soon as possible and may be different to this Accepted Article as a result of editing. Readers should obtain the VoR from the journal website shown below when it is published to ensure accuracy of information. The authors are responsible for the content of this Accepted Article.

To be cited as: Adv. Synth. Catal. 10.1002/adsc.202000875

Link to VoR: https://doi.org/10.1002/adsc.202000875 


\title{
A Nickel-Bisdiamine Porous Organic Polymer as Heterogeneous Chiral Catalyst for Asymmetric Michael Addition to Aliphatic Nitroalkenes
}

\author{
Mikkel B. Buendia, ${ }^{a}$ Søren Kegnæs, ${ }^{a *}$ Søren Kramer ${ }^{a *}$ \\ a Department of Chemistry, Technical University of Denmark, 2800 Kgs. Lyngby, Denmark \\ E-mail: skk@kemi.dtu.dk; E-mail: sokr@kemi.dtu.dk
}

Received: ((will be filled in by the editorial staff))

Supporting information for this article is available on the WWW under http://dx.doi.org/10.1002/adsc.201\#\#\#\#\#\#.

\begin{abstract}
We report a polystyrene-incorporated chiral nickel(II)-bisdiamine complex, which is accessible on gram-scale. This metal complex functions as a heterogeneous catalyst for the enantioselective Michael addition between malonates and aliphatic nitroalkenes, and it provides yields and enantioselectivities on par with homogeneous catalysts. Good functional group tolerance is reported for this reaction. Upon recycling, the catalyst achieves significantly higher TONs than previously reported. We demonstrate scalability to multigram-scale, compatibility with continuous flow production (4.43 gram), and application to the synthesis of the blockbuster drug Pregabalin. Finally, a new tandem reaction is disclosed.
\end{abstract}

Keywords: Asymmetric Catalysis; Heterogeneous Catalysis; Porous Organic Polymer; Michael Addition; Continuous Flow

The continuous demand for enantiopure compounds by the agrochemical, fragrance, and pharmaceutical industries necessitates more efficient syntheses of optically pure compounds. Asymmetric catalysis is one of the most powerful methods for obtaining enantiopure compounds, and homogeneous asymmetric catalysis has experienced astounding advancements over the past decades. ${ }^{[1]}$ However, for industrial purposes heterogeneous catalysis is generally preferred due to the ease of catalyst separation from the reaction medium and recycling. ${ }^{2]}$ For asymmetric catalysis, the benefits of the last feature are even more pronounced as the chiral ligands are often very expensive. Despite the development of several heterogenization strategies, covalent and non-covalent, it remains a challenge to obtain heterogeneous, recyclable catalysts which displays activity and enantioselectivity on par with the corresponding homogeneous catalysts. ${ }^{[3]}$

The asymmetric Michael addition reaction of malonates to nitroalkenes is a highly attractive reaction as the resulting products are easily converted into biologically active $\gamma$-amino acid derivatives, such as the pharmaceuticals Pregabalin and Rolipram. ${ }^{[4]}$
Several homogeneous catalytic systems have bee.. developed for this transformation ${ }^{[5]}$, however, successful reactions for nitroalkenes bearing aliphatic substituents are still rare. ${ }^{[6]}$ In contrast to reactions using homogeneous catalysts for the asymmetric Michael addition between malonates and nitroalkenes, reactions employing heterogeneous catalysts are much less investigated, especially for metal-based chiral catalysts. ${ }^{[4,7,8]}$ In 2012, two groups independently reported the first organometallic heterogeneous catalysts for this reaction. ${ }^{[4 a, 7 a]}$ Kobayashi and co-workers reported a polystyrenebound Pybox- $\mathrm{CaCl}_{2}$ catalyst, which displayed good enantioselectivity for aromatic nitroalkenes. ${ }^{[4 a]} \mathrm{Liu}$, $\mathrm{Li}$, and co-workers reported a chiral nickel(II)bisdiamine complex heterogenized by incorporation in periodic mesoporous organosilica. This catalyst system also provided good enantioselectivities for aromatic nitroalkenes. ${ }^{[7 \mathrm{a}]}$ In 2016, BelleminLaponnaz et al. incorporated a similar nickel complex into a self-supported polymerized catalyst, which showed good reusability and enantioselectivity for aromatic nitroalkenes. ${ }^{[7 \mathrm{~b}]}$ All the important advances mentioned above focus on aromatic nitroalkenes; aliphatic nitroalkenes were either unsuccessful or not included in these reports. Finally, in 2019, Kobayashi and co-workers reported the use of a catalyst consisting of a chiral nickel(II)-bisdiamine complex impregnated onto silica. ${ }^{[\mathrm{c}]}$ Good yields and enantioselectivities were achieved for a range of substrates including six aliphatic nitroalkenes without functional groups. Furthermore, recyclino experiments demonstrated loss of activity already in the second run caused by leaching of the chiral ligand due to the non-covalent support attachment.

Herein, we report a new heterogeneous catalyst for the asymmetric Michael addition of malonates to aliphatic nitroalkenes. The catalyst represents the first incorporation of a chiral nickel(II)-bisdiamine complex in a polystyrene porous organic polymer (POP). Due to the attachment of the ligands to the support by strong covalent bonds, no ligand leaching can take place. The heterogeneous nature of the catalyst makes it compatible with continuous flow 
production. Furthermore, a substrate scope investigation demonstrates - for the first time - good functional group tolerance for the challenging aliphatic nitroalkenes, manifested by high yields and enantioselectivities. Finally, a novel tandem reaction for a substrate bearing a pendant alkyne was discovered.

As part of our interest in asymmetric catalysis, catalysis with $3 \mathrm{~d}$ transition-metals, and heterogeneous catalysis, we set out to develop a highly efficient chiral heterogeneous metal catalyst for the challenging asymmetric Michael addition of malonates to aliphatic nitroalkenes. ${ }^{[9]} \mathrm{We}$ envisioned that the covalent incorporation of the ligands of a nickel(II)-bisdiamine catalyst into a polystyrene-POP would prevent undesired ligand leaching despite the fact that ligand dissociation is an integral part of the elucidated reaction mechanism for the homogeneous reaction. ${ }^{[5 \mathrm{~g}]}$ Recently, polystyrene-POP-based metal catalysts have received significant attention as their swelling properties allow for quasi-homogeneous catalyst behaviour while still facilitating easy catalyst separation and recycling. ${ }^{[9 \mathrm{~d}, 10]}$ The typical approach to chiral ligand-containing POP metal catalysts is polymerization of the ligands followed by postpolymerization metalation. This strategy has so far mostly focused on [ML] systems, typically with ligands within the PyBox, BINAP, and salen families (PyBox: 2,6-bis(4,5-dihydrooxazol-2-yl)pyridines; BINAP: $\quad 2,2$ '-bis(diphenylphosphino)-1,1'binaphthyl; salen:

N,N'bis(salicylidene)ethylenediamine)..$^{[3,9 \mathrm{~d}, 10]}$ To date, no examples of polystyrene-bound, co-polymerized $\left[\mathrm{ML}_{2}\right]$ catalysts bearing chiral ligands exist.

We initiated our study by preparing two polystyrene-POP catalysts by co-polymerization of vinyl-functionalized nickel(II)-bisdiamine complexes (Scheme 1). Thus, these materials can be classified as polystyrene-bound, co-polymerized $\left[\mathrm{ML}_{2}\right]$ catalysts bearing two chiral ligands. The complete synthesis route is short and simple, adding only a polymerization step in comparison to the synthesis of the corresponding homogeneous complexes. ${ }^{[11]}$ The direct synthesis of these POP-catalysts is notable as

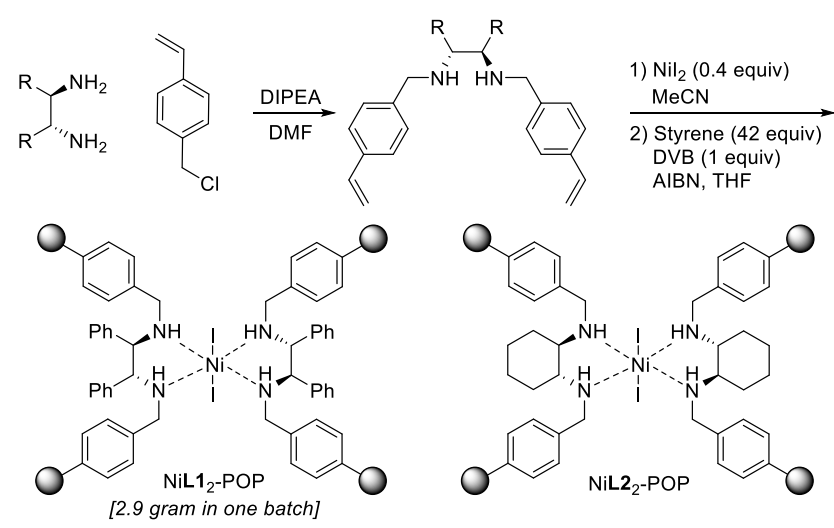

Scheme 1. Preparation of polystyrene-incorporated $\mathrm{NiL}_{2}-$ POPs. $(\mathrm{DVB}=$ divinylbenzene, $\mathrm{AIBN}=$ azobisisobutyronitrile, DIPEA = diisopropylethylamine).
Table 1. Evaluation of activity and enantioselectivity for POPs, containing different ligands, and the corresponding homogeneous catalysts.

\begin{tabular}{llll} 
Entry & Catalyst System & Yield $[\%]^{\mathrm{a})}$ & ee $[\%]^{\mathrm{b})}$ \\
\hline 1 & $\mathrm{NiL1}_{2}$-POP & 69 & 90 \\
2 & $\mathrm{NiL2}_{2}-\mathrm{POP}$ & 49 & 89 \\
$3^{\text {c) }}$ & $\mathrm{NiI}_{2} / \mathbf{L 1}$ & 68 & 90 \\
$4^{\text {c) }}$ & $\mathrm{NiI}_{2} / \mathbf{L 2}$ & 47 & 88 \\
5 & $\mathrm{NiI}_{2}$ & 0 & -
\end{tabular}

Reaction conditions: 1a $(0.25 \mathrm{mmol}), 2(0.35 \mathrm{mmol})$, and 1 mol\% catalyst in toluene $(1.0 \mathrm{~mL})$ at $60{ }^{\circ} \mathrm{C}$ for $5 \mathrm{~h}$. ${ }^{\text {a) }}$ Yield determined by ${ }^{1} \mathrm{H}$ NMR analysis using an internal standard. b) Enantiomeric excess determined by HPLC with a chiral stationary phase. ${ }^{\mathrm{c})} \mathrm{NiI}_{2}(1 \mathrm{~mol} \%)$ and ligand $(2.5 \mathrm{~mol} \%)$.

the synthesis of functionalized ligands suitable for POP-incorporation typically introduces several additional steps to already tedious synthetic routes. ${ }^{[3 a, 10]}$ In contrast, the straightforward route to $\mathrm{NiL}_{2}$-POPs allowed for easy preparation of almost 3 grams of catalyst material in one batch.

With the chiral heterogeneous catalysts in hand, we evaluated their performance in the asymmetric Michael addition of malonates to aliphatic nitroalkenes (Table 1). To allow for activity comparison, the reaction outcome was examined at partial conversion ( 5 hours) with low catalyst loading (1 mol\%). The comparable activities and enantioselectivities for polystyrene-bound and homogeneous complexes containing the same ligand highlight the quasi-homogeneous nature of the polystyrene-bound catalysts (entries 1-4). Although the rate-limiting steps for the homogeneous system involves ligand dissociation ${ }^{[5 \mathrm{~g}]}$, which incorporation into a polymer could potentially influence, we hypothesize that the extreme flexibility and quasihomogeneous nature of the polystyrene support in toluene makes the entropy-penalty for ligand dissociation very close to that of the homogeneous system. ${ }^{[12]}$ Both the polystyrene-bound and the homogeneous L1-complexes demonstrated improved activity compared to the L2-complexes. Thus, our study was continued with $\mathrm{NiL1} \mathbf{1}_{2}$-POP. ${ }^{[13]}$ A control experiment showed that $\mathrm{NiI}_{2}$ is not active withou diamine ligands (entry 5). Finally, evaluation of different malonates showed that methyl, ethyl, and iso-propyl malonates provided comparable activities, yields, and enantioselectivities, while the use of a tert-butyl malonate decreased the activity without affecting the enantioselectivity. ${ }^{[14]}$

Next, different ligand/support ratios and nickel halide salts were evaluated (Table 2). Simply polymerizing the vinyl-functionalized $\mathrm{NiL1} \mathbf{1}_{2}$ complex without styrene and DVB led to a catalyst displaying comparable activity but decreased enantioselectivity (entry 1). The addition of styrene and DVB to the 
Table 2. Evaluation of ligand/support ratios and counterion effects.

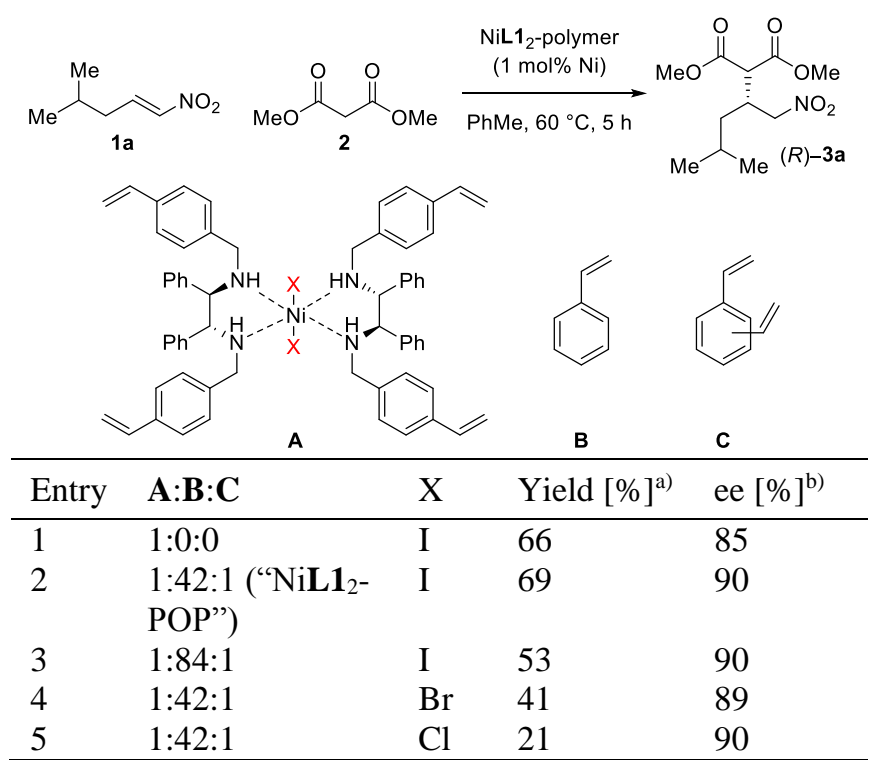

Reaction conditions: 1a $(0.25 \mathrm{mmol}), 2(0.35 \mathrm{mmol})$, and 1 mol\% catalyst in toluene $(1.0 \mathrm{~mL})$ at $60{ }^{\circ} \mathrm{C}$ for $5 \mathrm{~h}$. ${ }^{\text {a) }}$ Yield determined with ${ }^{1} \mathrm{H}$ NMR analysis using an internal standard. ${ }^{\text {b) }}$ Enantiomeric excess determined by HPLC with a chiral stationary phase.

polymerization improved the enantioinduction of the catalyst without affecting activity (entry 2). However, the use of additional styrene had a negative effect on the activity (entry 3 ). Notably, the halide counterion had significant impact on the activity as a decrease was observed when moving up in the periodic table (entries 4-5). The remaining study was performed with the NiL1 $\mathbf{1}_{2}$-POP catalyst in entry $2 .{ }^{[15]}$

Before further catalytic evaluations were performed, the optimized $\mathrm{NiL1}_{2}$-POP was characterized ${ }^{[11]}{ }^{13} \mathrm{C}-{ }^{1} \mathrm{H}$ CP/MAS NMR spectroscopy of the catalyst material in the solid state clearly showed ligand incorporation into the polystyrene backbone. Transmission electron microscopy (TEM) confirmed the absence of metal nanoparticles in the material, and energy-dispersive X-ray spectroscopy proved the presence of both nickel and iodide. The nickel content was determined to be 1.07 wt.\% by inductively coupled plasma optical emission spectroscopy (ICP-OES). Thermogravimetric analysis indicated that the $\mathrm{NiL1}_{2}-\mathrm{POP}$ is stable in air up to $300{ }^{\circ} \mathrm{C}$. Scanning electron microscopy (SEM) showed that $\mathrm{NiL1}_{2}$-POP consisted of both hollow sheets, in the size range $10 \mu \mathrm{m}$ to $50 \mu \mathrm{m}$, and spheres with a broad size distribution ranging from $50 \mathrm{~nm}$ to a $1 \mu \mathrm{m}$. The $\mathrm{NiL1}_{2}$-POP had a negligible surface area and pore volume of $24 \mathrm{~m}^{2} / \mathrm{g}$ and $0.049 \mathrm{~cm}^{3} / \mathrm{g}$, respectively. These results suggest that the high catalytic activity of $\mathrm{NiL1}_{2}$-POP is caused by its swelling properties. In fact, during catalysis, the reaction mixture is a clear solution thus indicating the quasi-homogeneous nature of the catalysis. ${ }^{[11]}$ Finally, we tested nickel leaching during a catalytic reaction by ICP-OES. The nickel content in the liquid phase

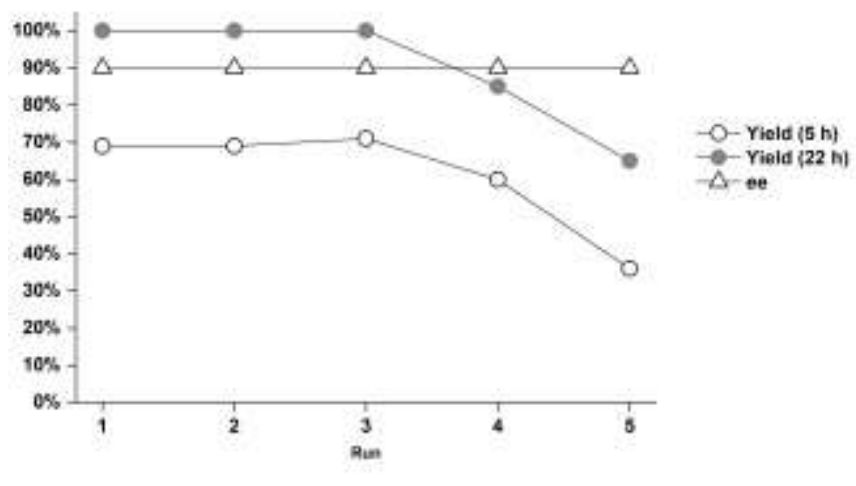

Figure 1. Recycling experiments including activity tests for the reaction between $\mathbf{1 a}$ and $\mathbf{2}$. Reactions conditions as Table 2, entry 2 .

after 22 hours corresponded to $0.7 \%$ of the original nickel content in the catalyst material, hence very limited nickel leaching is taking place. ${ }^{[16]}$

With the optimized catalyst in hand, we investigated the recyclability of the heterogeneous chiral catalyst. At the end of a reaction, $\mathrm{NiL1} \mathbf{1}_{2}-\mathrm{POP}$ is easily recovered by centrifugation after addition of ether/hexane. Using this method, five consecutive runs were performed with the same catalyst material (Figure 1). For all five runs, the enantioselectivity remained constant at $90 \%$ ee. Furthermore, the $\mathrm{NiL1}_{2}$-POP displayed no loss of activity during the first three runs (yields after 5 hours). Although the activity started to decrease after three consecutive reactions, a turnover number (TON) of 450 was obtained after five runs. This TON is almost five times higher than any previously reported TON. ${ }^{[17]}$ TEM images after the fifth consecutive run did not show any metal nanoparticle formation. In combination with the very limited nickel leaching, the origin of the deactivation is not clear at this point.

Encouraged by the high performance of $\mathrm{NiL1}_{2}$ POP as heterogeneous catalyst, in terms of yield, enantioselectivity and TON, we continued by investigating the substrate scope for the nitroalkenes. So far, aliphatic nitroalkenes bearing functional groups have not been reported for the asymmetric Michael addition of malonates. ${ }^{[5,6,7]}$ Accordingly, we decided to examine both the tolerance to different carbon scaffolds and functional groups in order to improve the utility of this reaction (Table 3). First, the sensitivity to length and sterical hindrance of th alkyl substituent was evaluated. The reaction efficiency was not affected by increasing the length of an unbranched alkyl moiety (3b-3d). Also, introduction of the bulkier iso-propyl group did not affect the reaction outcome significantly, while the use of a cyclohexyl-bearing substrate led to a small decrease in yield (3e-3f). The sterically highly challenging tert-butyl group was tolerated but led to lower yield and enantioselectivity (3g). Importantly, the reaction was found to tolerate a broad range of functional groups including sensitive functional 


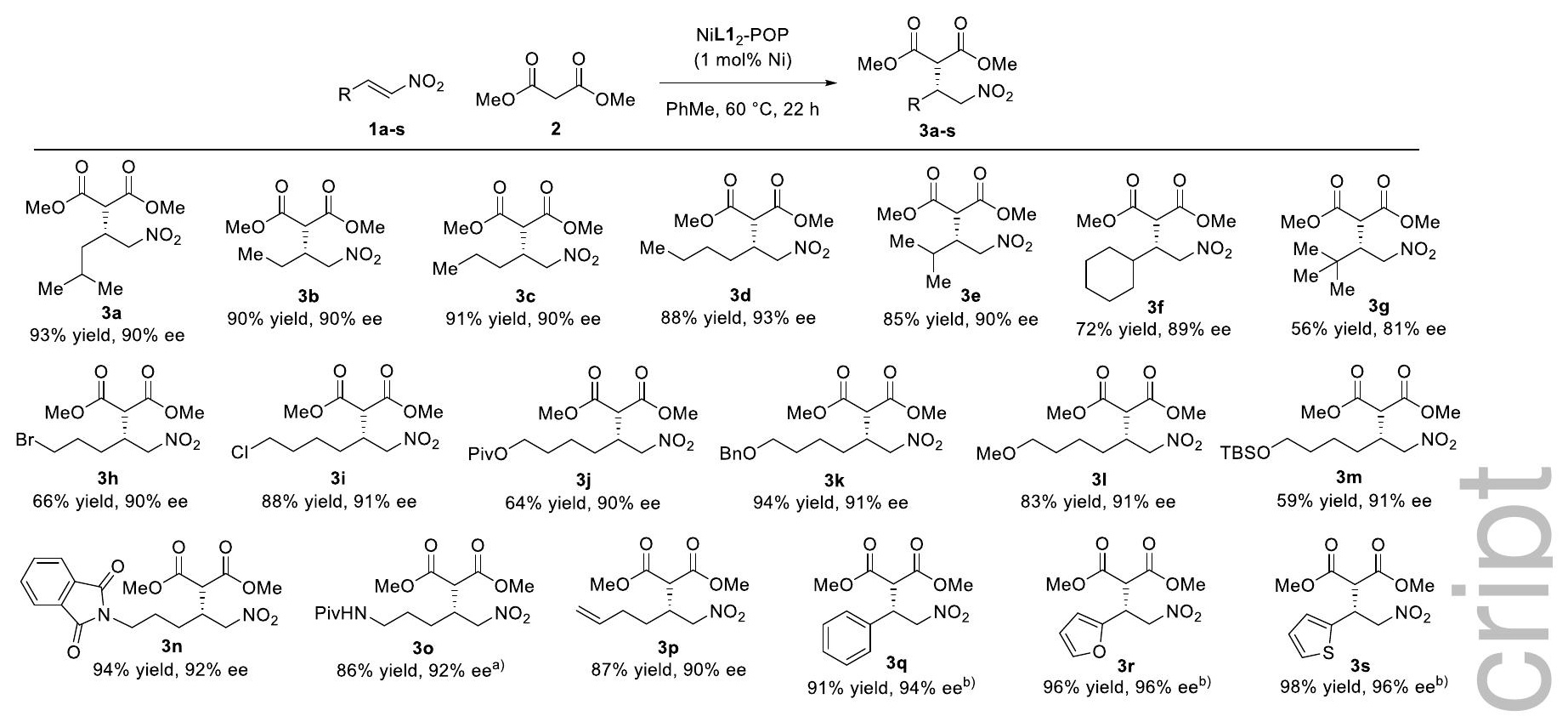

Table 3. Substrate scope for nitroalkenes. The listed yields are isolated yield of purified product. Reaction conditions: 1 $(0.375 \mathrm{mmol}), 2(0.525 \mathrm{mmol})$, and $1 \mathrm{~mol} \%$ catalyst in toluene $(1.5 \mathrm{~mL})$ at $60{ }^{\circ} \mathrm{C}$ for $22 \mathrm{~h}$. Enantiomeric excess determined by HPLC with a chiral stationary phase. ${ }^{\text {a) }} 2$ mol\% NiL1 2 -POP, 72 h. ${ }^{\text {b) }}$ Room temperature.

groups, and all these substrates afforded high enantioselectivities $(\geq 90 \%$ ee). Primary alkyl bromides and chlorides were tolerated (3h-3i), as

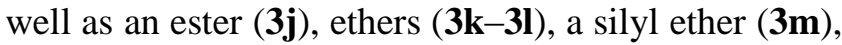
a phthalimide-protected amine (3n), an amide (3o), and a terminal alkene (3p). The absolute stereochemistry was assigned by X-ray crystallographic analysis of $\mathbf{3 n}$ (Figure 2). ${ }^{[11]}$ Three (hetero)aromatic nitroalkenes were also included in the substrate scope investigation in order to illustrate the generality of the developed catalyst $(\mathbf{3 q}-\mathbf{3 s})$.

Next, the scalability of the developed method was examined. A gram-scale reaction was performed using $1 \mathrm{~mol} \%$ of $\mathrm{NiL1}_{2}$-POP in undried toluene and under air (Scheme 2a). Pleasingly, using this protocol, the desired product was obtained in a quantitative yield (2.20 gram) and with $90 \%$ ee. Subsequently, we proceeded to evaluate the compatibility of the heterogeneous catalyst with a continuous flow setup (Scheme 2b). ${ }^{[18]}$ The adaptation proceeded smoothly, and the catalyst showed good stability for more than five continuous days of operation which produced

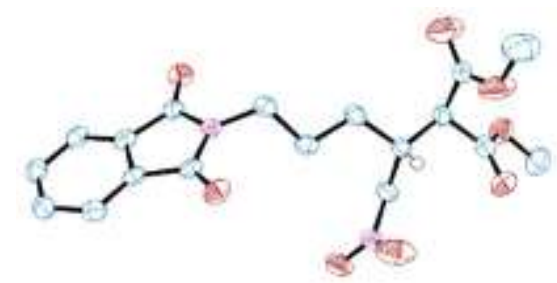

Figure 2. X-ray crystal structure of 3n (ellipsoids are shown at $50 \%$ probability, and most hydrogens are omitted for clarity). ${ }^{[19]}$
4.43 grams of $(R)-3 \mathbf{a}$ in $90 \%$ ee. Again, the catalyst efficiency was remarkably high, and a TON of 173 was obtained which is almost three times higher than previously reported. ${ }^{[20]}$

The opposite enantiomer of $\mathrm{NiL1} \mathbf{1}_{2}$-POP is easily accessible using the same synthesis route (Scheme 1), As expected, the use of $\mathrm{Ni}(\text { ent-L1 })_{2}$-POP afforded the opposite enantiomer of product in essentially

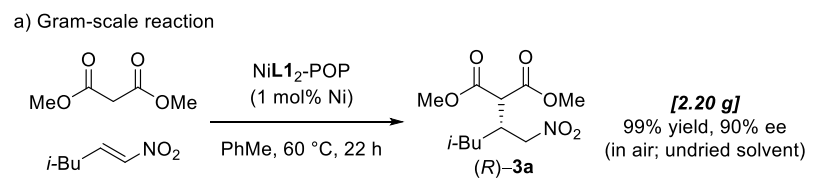

b) Application in continuous flow for multigram-scale production

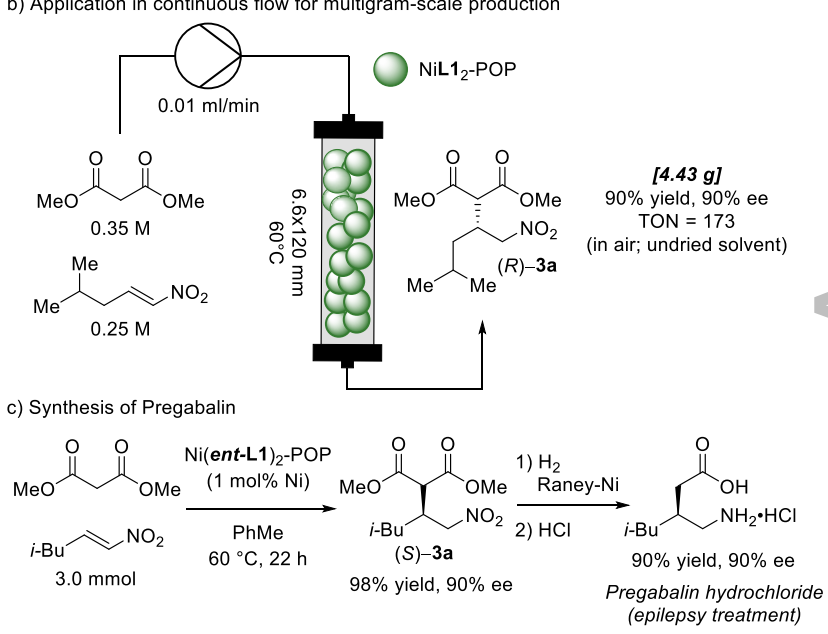

Scheme 2. Application of the developed method (a) on gram-scale, (b) in continuous flow production, and (c) for enantioselective synthesis of the pharmaceutical Pregabalin. 
identical yield and enantioselectivity compared to NiL1 2 -POP (Scheme 2c). In just two steps from this product, $(S)-\mathbf{3 a}$, the blockbuster drug Pregabalin was synthesized in $90 \%$ yield and $90 \%$ ee, thus highlighting the relevance of the reaction and products for medicinal chemistry. ${ }^{[21,22]}$

Finally, during the investigation of the substrate scope, an unprecedented tandem reaction was discovered for a substrate bearing a pendant alkyne (1t) (Scheme 3). The formal [4+1] annulation affords $98 \%$ yield of the enantioenriched exomethylenecyclopentane 3t. The reaction proceeds by an initial enantioselective Michael addition followed by a Conia-ene reaction. ${ }^{[23]}$ Build-up of the intermediate from the Michael addition is observed during the reaction suggesting that the Conia-ene cyclization is the slowest step. Furthermore, no cyclization of the isolated intermediate was observed in the absence of $\mathrm{NiL \mathbf {1 } _ { 2 }}$-POP indicating that the nickel complex is responsible for catalyzing both steps. It is rare that both steps of a tandem Michael addition/Conia-ene sequence is catalyzed by the same catalyst. ${ }^{[23]}$ Notably, there are no previous reports on racemic or enantioselective [4+1] annulation between malonates and alkyne-bearing nitroalkenes. ${ }^{[24]}$

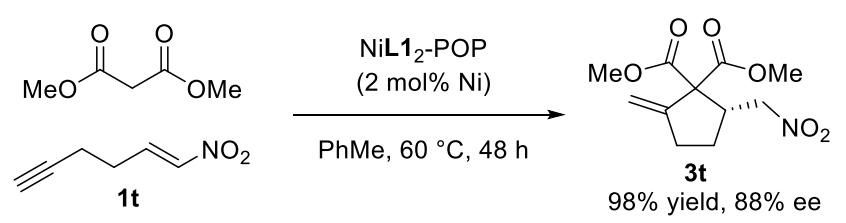

Scheme 3. Discovery of a new tandem reaction. Both steps of the formal $[4+1]$ annulation are catalyzed by $\mathrm{Ni} \mathbf{L 1}_{2}-$ POP.

In conclusion, we have prepared a polystyreneincorporated chiral nickel(II)-bisdiamine metal complex, which can be used as a heterogeneous catalyst for the challenging asymmetric Michael addition of malonates to aliphatic nitroalkenes. The catalyst provides high yields and enantioselectivities for this reaction. Our protocol was found to tolerate a broad range of functional groups, including sensitive groups. Importantly, the catalyst displayed good recyclability, and a TON almost five times higher than previously reported was obtained by recycling in batch. Furthermore, the reaction is scalable to multigram-scale both in batch and in a continuous flow setup. The relevance of the transformation and products for medicinal chemistry was highlighted with the synthesis of Pregabalin. Finally, we have discovered an unprecedented tandem reaction between malonates and alkyne-functionalized nitroalkenes leading to an enantioenriched exomethylenecyclopentane. Overall, we have reported a heterogeneous catalyst displaying activity and enantioselectivity on par with the homogeneous catalysts, and we have illustrated advantages of the heterogeneous catalyst such as easy catalyst separation and recycling as well as application in continuous flow production.

\section{Experimental Section}

In air, NiL1 2 -POP (23.4 mg, 1.0 mol\%), dimethyl malonate $(60.0 \mu \mathrm{L}, 0.575 \mathrm{mmol})$, nitroalkene $(0.375$ mmol) and toluene $(1.5 \mathrm{~mL})$ were added to a $4 \mathrm{~mL}$ vial. The vial was capped with a PTFE-lined septum cap and the reaction stirred for 22 hours at $60{ }^{\circ} \mathrm{C}$. After cooling to room temperature, the reaction mixture was directly purified by flash chromatography.

\section{Acknowledgements}

The authors are grateful for funding from the Independen: Research Fund Denmark (grant no. 6111-00237), from Villum Foundation (grant no. 13158), and from Haldor Topsфe A/S. S. Kramer is deeply appreciative of generous financial support from the Lundbeck Foundation (grant no. R250-2017-1292). Th. authors thank David B. Christensen for assistance with TEM, Dr. Farnoosh Goodarzi for assistance with SEM, Dr. Mariusz Kubus for assistance with X-ray crystallography, and Dr. Kasper Enemark-Rasmussen at the DTU NMR center supported by the Villum foundation for aid with solid-state NMR.

\section{References}

[1] I. Ojima, Catalytic Asymmetric Synthesis, Third Edition, Wiley-VCH, Weinheim, 2010.

[2] J. Hagen, Industrial Catalysis: A Practical Approach, Third Edition, Wiley-VCH, Weinheim, 2015.

[3] a) K. Ding, Y. Uozumi, Handbook of Asymmetric Heterogeneous Catalysis, Wiley-VCH, Weinhein, 2008; b) H. U. Blaser, H. J. Federsel, Asymmetric Catalysis on Industrial Scale: Challenges, Approache. and Solutions: Second Edition, Wiley-VCH, Weinheim, 2010.

[4] a) T. Tsubogo, Y. Yamashita, S. Kobayashi, Chem. Eur. J. 2012, 18, 13624-13628; b) T. Tsubogo, H. Oyamada, S. Kobayashi, Nature 2015, 520, 329-332.

[5] a) T. Okino, Y. Hoashi, Y. Takemoto, J. Am. Chem. Soc. 2003, 125, 12672-12673; b) H. Li, Y. Wang, L. Tang, L. Deng, J. Am. Chem. Soc. 2004, 126, 99069907; c) D. A. Evans, D. Seidel, J. Am. Chem. Soc. 2005, 127, 9958-9959; d) Okino, Y. Hoashi, T Furukawa, X. Xu, Y. Takemoto, J. Am. Chem. Soc. 2005, 127, 119-125; e) J. Ye, D. J. Dixon, P. S. Hynes, Chem. Commun. 2005, 4481-4483; f) M. Terada, H Ube, Y. Yaguchi, J. Am. Chem. Soc. 2006, 128, 14541455; g) D. A. Evans, S. Mito, D. Seidel, J. Am. Chem. Soc. 2007, 129, 11583-11592; h) Q. Zhu, H. Huang, D. Shi, Z. Shen, C. Xia, Org. Lett. 2009, 11, 4536-4539; i) K. Wilckens, M.-A. Duhs, D. Lentz, C. Czekelius, Eur. J. Org. Chem. 2011, 2011, 5441-5446; j) T. Mukherjee, C. Ganzmann, N. Bhuvanesh, J. A. Gladysz, Organometallics 2014, 33, 6723-6737; k) K. G. Lewis, S. K. Ghosh, N. Bhuvanesh, J. A. Gladysz, ACS Cent. Sci. 2015, 1, 50-56; 1) S. K. Ghosh, C. Ganzmann, N. Bhuvanesh, J. A. Gladysz, Angew. Chem. Int. Ed. 2016, 55, 4356-4360; m) D. Bécart, V. Diemer, A. Salaün, M. 
Oiarbide, Y. R. Nelli, B. Kauffmann, L. Fischer, C. Palomo, G. Guichard, J. Am. Chem. Soc. 2017, 139, 12524-12532.

[6] K. Zheng, X. Liu, X. Feng, Chem. Rev. 2018, 118, 7586-7656.

[7] a) K. Liu, R. Jin, T. Cheng, X. Xu, F. Gao, G. Liu, H. Li, Chem. Eur. J. 2012, 18, 15546-15553; b) D. Bissessar, T. Achard, S. Bellemin-Laponnaz, Adv. Synth. Catal. 2016, 358, 1982-1988; c) H. Ishitani, K. Kanai, W. J. Yoo, T. Yoshida, S. Kobayashi, Angew. Chem. Int. Ed. 2019, 58, 13313-13317.

[8] For examples which do not use a metal catalyst, see: a) K. A. Fredriksen, T. E. Kristensen, T. Hansen, Beilstein J. Org. Chem. 2012, 8, 1126-1133; b) A. M. Goldys, M. G. Nuñez, D. J. Dixon, Org. Lett. 2014, 16, 6295-6297; c) X. Xu, T. Cheng, X. Liu, J. Xu, R. Jin, G. Liu, ACS Catal. 2014, 4, 2137-2142; d) I. Billault, R. Launez, M.-C. Scherrmann, RSC. Adv. 2015, 5, 29386-29390; e) M. S. Ullah, S. Itsuno, ACS Omega, 2018, 3, 45734582 .

[9] a) F. Wang, J. Mielby, F. Richter, G. Wang, G. Prieto, T. Kasama, C. Weidenthaler, H.-J. Bongard, S. Kegnæs, A. Fürstner, F. Schüth, Angew. Chem. Int. Ed. 2014, 53, 8645-8648; b) S. Kramer, G. C. Fu, J. Am. Chem. Soc. 2015, 137, 3803-3806; c) S. Kramer, F. Hejjo, K. H. Rasmussen, S. Kegnæs, ACS Catal. 2018, 8, 754-759; d) S. Kramer, N. R. Bennedsen, S. Kegnæs, ACS Catal. 2018, 8, 6961-6982; e) N. R. Bennedsen, R. M. Mortensen, S. Kramer, S. Kegnæs, J. Catal. 2019, 371, 153-160; f) J. Huang, M. Hong, C.-C. Wang, S. Kramer, G.-Q. Lin, X.-W. Sun, J. Org. Chem. 2018, 83, 12838-12846; g) S. Kramer, Org. Lett. 2019, 21, 6569; h) J. Himmelstrup, M. Buendia, X.-W. Sun, S. Kramer, Chem. Commun. 2019, 55, 12988-12991.

[10] For reviews, see: a) C. A. McNamara, M. J. Dixon, M. Bradley, Chem. Rev. 2002, 102, 3275-3300; b) P. Kaur, J. T. Hupp, S. T. Nguyen, ACS Catal. 2011, 1, 819835; c) M. Gruttadauria, F. Giacalone, Catalytic Methods in Asymmetric Synthesis: Advanced Materials, Techniques, and Applications, John Wiley and Sons, Hoboken, 2011; d) Q. Sun, Z. Dai, X. Meng, F.-S. Xiao, Chem. Soc. Rev. 2015, 44, 6018-6034; e) L. Tan, B. Tan, Chem. Soc. Rev. 2017, 46, 3322-3356; f) B. Altava, M. I. Burguete, E. García-Verdugo, S. V Luis, Chem. Soc. Rev. 2018, 47, 2722-2771.

[11] See supporting information for details.

[12] For previous examples where polymer-supported catalysts display comparable activity to the corresponding homogeneous catalysts, see: a) T. S. Reger, K. D. Janda, J. Am. Chem. Soc. 2000, 122, 6929-6934; b) Q. Sun, Z. Dai, X. Meng, F.-S. Xiao, Chem. Mater. 2017, 29, 5720-5726; c) B. Altava, M. I. Burguete, E. Garcia-Verdugo, S. V. Luis, Chem. Soc. Rev. 2018, 47, 2722-2771.

[13] A $\mathrm{NiL1}_{2}$-POP made by post-polymerization metalation led to essentially identical activity and enantioselectivity (5 h: $65 \%$ yield, $90 \%$ ee; see Table S3). The similar activities and enantioselectivities for the homogeneous catalyst, the $\mathrm{NiL1} \mathbf{1}_{2}-\mathrm{POP}$, and the post-polymerization metalation catalyst also indicates that that a $\mathrm{Ni}$ :diamine ratio of $1: 2$ is maintained during polymerization for $\mathrm{NiL1}_{2}-\mathrm{POP}$. See ref $5 \mathrm{~g}$.

[14] Results for the different malonates after $22 \mathrm{~h}$ (NMR yields): Methyl: $>99 \%$ yield, $90 \%$ ee; ethyl: $>99 \%$ yield, $90 \%$ ee; iso-propyl: $>99 \%$ yield, $90 \%$ ee; tertbutyl: $80 \%$ yield, $90 \%$ ee. For more details, see Table S4.

[15] The use of ethyl acetate or THF as solvent, under the reaction conditions in Table 2, entry 2, provided inferior results after 5 h: EtOAc, $31 \%$ yield, $89 \%$ ee; THF, $45 \%$ yield, $81 \%$ ee.

[16] To ensure that leaching of homogeneous species are not responsible for the catalytic activity, a hot filtration test was performed. After filtration of a reaction mixture at $25 \%$ conversion, no further catalytic activity was observed in the liquid phase, thus indicating that the catalytic activity is not caused by leached homogeneous species (See Figure S8).

[17] The best previously reported TON for heterogeneous catalysts with malonates and aliphatic nitroalkenes in batch is 95 . See ref. $7 \mathrm{c}$.

[18] a) D. Webb, T. F. Jamison, Chem. Sci. 2010, 1, 675680 ; b) R. L. Hartman, J. P. McMullen, K. F. Jensen, Angew. Chem. Int. Ed. 2011, 50, 7502-7519; c) J.-I. Yoshida, H. Kim, A. Nagaki, ChemSusChem, 2011, 4, 331-340; d) J. C. Pastre, D. L. Browne, S. V. Ley, Chem. Soc. Rev. 2013, 42, 8849-8869.

[19] CCDC-2017002 contains the supplementary crystallographic data for this paper. These data can $b_{2}$ obtained free of charge from The Cambridge Crystallographic Data Centre www.ccdc.cam.ac.uk/data_request/cif.

[20] The best previously reported TON for heterogeneous catalysts with malonates and aliphatic nitroalkenes in continuous flow is 63 (producing $\approx 250 \mathrm{mg} ; 87 \%$ ee). See ref. $7 c$.

[21] In 2018, Pregabalin had the $16^{\text {th }}$ highest retail sales of all drugs. (https://njardarson.lab.arizona.edu/sites/njardarson.lab. arizona.edu/files/2018Top200PharmaceuticalRetailSale sPosterLowResFinalV2.pdf (accessed July 2020).

[22] The absolute stereochemistry of product $(S)-\mathbf{3 a}$ was determined by comparison of the optical rotation of Pregabalin hydrochloride with literature: H.-J. Yu, C Shao, Z. Cui, C.-G. Feng, and G.-Q. Lin, Chem. Eur. J. 2012, 18, 13274-13278.

[23] D. Hack, M. Blümel, P. Chauhan, A. R. Philipps, D. Enders, Chem. Soc. Rev. 2015, 44, 6059-6093.

[24] For a related formal [4+1] annulation between pyrazolones and arylacetylene-bearing nitroalkenes using dual silver and organocatalysis, see: D. Hack, A. B. Dürr, K. Deckers, P. Hauhan, N. Seling, L. Rübenach, L Mertens, G. Raabe, F. Schoenebeck, D. Enders, Angew. Chem. Int. Ed. 2016, 55, 1797-1800. 


\section{UPDATE}

A Nickel-Bisdiamine Porous Organic Polymer as Heterogeneous Chiral Catalyst for Asymmetric Michael Addition to Aliphatic Nitroalkenes

Adv. Synth. Catal. Year, Volume, Page - Page

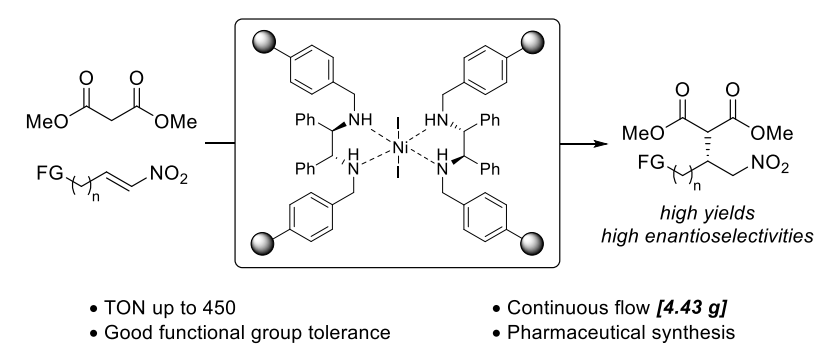

Mikkel B. Buendia, Søren Kegnæs, * Søren - Good functional group tolerance 